Received: $\quad 2018.01 .26$

Accepted: 2018.02.15

Published: 2018.07.16

\title{
The Effects of Ludartin on Cell Proliferation, Cell Migration, Cell Cycle Arrest and Apoptosis Are Associated with Upregulation of p21WAF1 in Saos-2 Osteosarcoma Cells In Vitro
}

Authors' Contribution: Study Design A

Data Collection B Statistical Analysis C Data Interpretation D Manuscript Preparation E Literature Search F Funds Collection $C$

\author{
BCDEF 1,2 Shuang-li Zhang \\ BDEF 3 Bao-lin Li \\ BEF 3 Wei Li \\ BCD 4 Ming Lu \\ $B C D 5$ Lin-ying Ni \\ BC 6 Hui-li Ma
}

ACDEFG 1,3 Qing-gang Meng
1 Harbin Medical University, Harbin, Heilongjiang, P.R. China

2 Department of Oncological Surgery, The First Hospital of Qiqihaer City, Qiqihaer, Heilongjiang, P.R. China

3 Department of Orthopedics, The First Hospital of Harbin, Harbin, Heilongjiang, P.R. China

4 Department of Surgical Oncology, The First Hospital of Qiqihaer City, Qiqihaer, Heilongjiang, P.R. China

5 Department of Orthopedics, Harbin Medical University, Cancer Hospital, Harbin, Heilongiiang, P.R. China

6 Department of Orthopedics, Binzhou Medical University Hospital, Binzhou, Shandong, P.R. China
Corresponding Author: Source of support:

Background: The aim of this study was investigate the effects of the sesquiterpene lactone, ludartin, on cell proliferation, cell migration, apoptosis, and the cell cycle in osteosarcoma cell lines, compared with a normal osteoblast cell line.

Material/Methods

Osteosarcoma cell lines, MG-63 Saos-2 U-2OS, T1-73 143B, and HOS, and normal hFOB 1.19 osteoblasts, were cultured and treated with increasing doses of ludartin, The MTT colorimetric assay was used to measure cell metabolic activity and viability. Apoptosis was studied by fluorescence-activated cell sorting (FACS) using 4',6-diamidino-2-phenylindole (DAPI) nuclear staining and Annexin-V/propidium iodide (PI) staining. Cell cycle was studied using flow cytometry. Cell migration and invasion were studied using wound healing and Boyden chamber assays. Protein expression was measured by Western blotting.

Results: Ludartin inhibited cell viability, cell migration, cell proliferation, and increased cell apoptosis, in all osteosarcoma cell lines, with an $\mathrm{IC}_{50}$ dose ranging from 15-30 $\mu \mathrm{M}$. The greatest effects were on the Saso-2 osteosarcoma cells, with an $\mathrm{IC}_{50}$ of $15 \mu \mathrm{M}$. However, ludartin showed minor cytotoxic effects of the normal hFOB 1.19 osteoblasts $\left(\mathrm{IC}_{50}>100 \mu \mathrm{M}\right)$. Ludartin exerted its anti-proliferative effects on Saos-2 cells via induction of apoptosis and cell cycle arrest at the G2/M checkpoint, associated with reduced expression of Cdc25c (Ser216), Cdc25c, pCdc2 (Tyr15), and Cdc2 and increased expression of p21WAF1. Ludartin inhibited cell migration and invasion of the Saos- 2 cells.

Conclusions: The dose-dependent effects of ludartin on cell proliferation, migration, apoptosis, cell cycle arrest at the G2/M checkpoint involved p21WAFI in Saos-2 osteosarcoma cells.

MeSH Keywords: $\quad$ Apoptosis • Cell Cycle • Cell Migration Assays • Osteosarcoma

Full-text PDF: https://www.medscimonit.com/abstract/index/idArt/909193

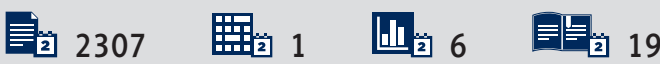




\section{Background}

Osteosarcoma is the most common primary bone tumor in adolescents and children, with more than $70 \%$ of cases occurring in the age group of between 10-25 years [1]. In cases of childhood osteosarcoma, a 35\% mortality rate has been reported, despite the use of available treatment strategies [2,3]. In adult patients with primary osteosarcoma, chemotherapeutic agents are often ineffective because of the development of chemoresistance. Although there has been increasing progress in the diagnosis and treatment of osteosarcoma, including surgery, chemotherapy, and radiation therapy, because of the development of early metastasis and resistance to chemotherapeutic agents, there is a need to discover novel diagnostic and prognostic biomarkers and supplement current treatments. Ongoing studies are being conducted to understand the development and progression of osteosarcoma and potential diagnostic and prognostic biomarkers [4-6]. However, the discovery of new molecules with the potential to inhibit osteosarcoma and the development of new treatment strategies are still needed.

In Chinese herbal medicine, natural or herbal derived medicines have shown promising results as chemotherapeutic agents for different types of cancers, and in China, more than half of the existing anticancer drugs are derived from natural products $[7,8]$. Ludartin is a sesquiterpene lactone that exhibits a wide range of pharmacological properties including, cytotoxic, anticancer, and bactericidal activities $[9,10]$. However, the anticancer activity of ludartin against the osteosarcoma has not been previously evaluated.

The aim of this in vitro study was to investigate the effects of the sesquiterpene lactone, ludartin, on cell proliferation, cell migration, apoptosis, and the cell cycle in osteosarcoma cell lines, compared with a normal osteoblast cell line.

\section{Material and Methods}

\section{Cell culture}

Osteosarcoma cell lines included MG-63 Saos-2 U-20S, T173 143B, HOS, and normal osteoblast cells, hFOB 1.19 were purchased from the American Type Culture Collection (ATCC) (Rockville, MD, USA). The cells were cultured in Dulbecco's modified Eagle's medium (DMEM) containing 10\% fetal bovine serum (FBS) and antibiotics and maintained in a humidified atmosphere including $5 \% \mathrm{CO}_{2}$ and maintained at a temperature of $37^{\circ} \mathrm{C}$.

\section{MTT assay}

The proliferation rate of osteosarcoma and normal cells were analyzed by the MTT assay. The cells were cultured at a density of $3 \times 10^{6}$ cells per $\mathrm{ml}$ in a 96 -well plate, and cultured for $24 \mathrm{~h}$ at $37^{\circ} \mathrm{C}$. Incubation of the cells was performed for $48 \mathrm{~h}$ at a concentration of between $0-100 \mu \mathrm{M}$ of ludartin in a humidified atmosphere of $5 \% \mathrm{CO}_{2}$ at a temperature of $37^{\circ} \mathrm{C}$. A volume of $150 \mu \mathrm{l}$ of MTT solution $(5 \mathrm{mg} / \mathrm{ml}$ ) was added to each well of the 96-well plate and incubated for four more hours. The supernatant was decanted from each well. The formazan crystals that formed were dissolved by the addition of $150 \mu$ of dimethyl sulfoxide (DMSO). The absorbance for each of the wells was recorded at $465 \mathrm{~nm}$ using a spectrophotometer.

\section{Apoptosis analysis by flow cytometry}

After $48 \mathrm{~h}$ of incubation, the cells were incubated with $0,7.5$, 15 , and $30 \mu \mathrm{M}$ concentrations of ludartin. The Saos- 2 cells were selected, collected, and washed with phosphate buffered saline (PBS). The cells were then stained using 4',6-diamidino-2-phenylindole (DAPI) nuclear staining and apoptosis was detected by fluorescence microscopy, as previously reported [11]. For measurement of apoptotic cell populations, the ludartin-treated cells were then suspended in binding buffer at a density of $3 \times 10^{6}$ cells per $\mathrm{ml}$ followed by staining with $5 \mu$ l of Annexin-V fluorescein isothiocyanate (FITC) and $5 \mu \mathrm{l}$ propidium iodide (PI). The cell suspension was incubated in the dark at room temperature for $25 \mathrm{~min}$. Analysis of cell apoptosis was carried out using a BD FACSCalibur ${ }^{\mathrm{TM}}$ flow cytometer (BD Biosciences, NJ, USA).

\section{Cell cycle analysis}

To determine the distribution of cells in each phase of the cell cycle, the ludartin-treated Saso-2 osteosarcoma cells were collected and washed with PBS, fixed with ethanol (70\%) for about an hour, and then washed again with PBS. The cells were resuspended in a solution of $\mathrm{PI}(50 \mu \mathrm{l} / \mathrm{ml})$ and RNase $1(250 \mu \mathrm{g} / \mathrm{ml})$, followed by incubation for $30 \mathrm{~min}$ at room temperature. Cell cycle was investigated using the fluorescence of Annexin-V and PI using FC500 fluorescence-activated cell sorting (FACS) caterplus flow cytometry (Beckman Coulter, CA, USA) at an excitation wavelength of $488 \mathrm{~nm}$ and emission wavelengths of 525 and $625 \mathrm{~nm}$, respectively using 10,000 cells/group.

\section{Cell migration assay}

The cell migration capacity of ludartin-treated osteosarcoma cells was examined using a wound healing assay. Briefly, $5 \times 10^{4}$ cells/well were cultured in 96-well plates and were incubated overnight at $37^{\circ} \mathrm{C}$ to allow the cells to adhere. A wound was then created with a scratch using a sterile pipette tip after the cells reached confluence. The cells were then washed with PBS to clear the detached cells. The cells were monitored after an interval of $20 \mathrm{~h}$ interval and photographed. The invasive properties of the ludartin-treated Saso-2 cells were determined 
using a Boyden Chamber assay as previously described [12]. Briefly, the Boyden Chamber assay used a plastic chamber containing a porous membrane, suspended over a larger well containing medium or chemoattractant and the cells that migrate through the pores to the other side of the membrane and were stained and counted.

\section{Western blot analysis}

The cells were incubated for $48 \mathrm{~h}$ with $25 \mathrm{~nm}$ concentration of ludartin, harvested, rinsed in cold PBS and lysed in cold lysis buffer for $45 \mathrm{~min}$. The cell lysates were then centrifuged at $15,000 \times g$ for $15 \mathrm{~min}$ at a temperature of $4^{\circ} \mathrm{C}$, and the supernatant was collected. A Bicinchoninic Acid Protein Assay kit (Pierce, Rockford, IL, USA) was used to measure the concentration of proteins according to the manufacturer's instructions. Protein samples $(50 \mu \mathrm{g})$ were isolated on $12 \%$ sodium dodecyl sulphate-polyacrylamide gels by electrophoresis. The proteins were transferred onto polyvinylidene difluoride (PVDF) membranes, blocked using $5 \%$ bovine serum albumin (BSA) and Trisbuffered saline with Tween ${ }^{\circledR} 20$ (TBST) buffer using a semidry transfer system (Bio-Rad Laboratories, Hercules, CA, USA).

The membrane was then incubated overnight at $4^{\circ} \mathrm{C}$ using the dilution of 1: 1,000, with the following primary antibodies to: matrix metallopeptidase (MMP)-9 (Cat. No. sc-176046); MMP-2 (Cat. No. sc-176407); Cdc25c (Cat. No. sc-327); Chk2 (Cat. No. sc-56296), p-Cdc2 (Tyr15) (Cat. No. sc-12340); Cdc2 (Cat. No. sc-54); cyclin B1 (Cat. No. sc-70898); p27 (Cat. No. sc-1641); p21 (p21WAFI) (Cat. No. sc-122305); p53 (Cat. No. sc-47698); actin (Cat. No. sc-58673); GAPDH (Cat. No. sc-47724) (Santa Cruz Biotechnology Inc., Dallas TX, USA).
Table 1. Antiproliferative effects of Ludartin on osteosarcoma and normal cell lines determined by MTT assay and expressed as $\mathrm{IC}_{50}(\mu \mathrm{M})$.

\begin{tabular}{|cc|}
\hline Cell lines & $\mathbf{I C}_{\mathbf{5 0}}(\boldsymbol{\mu} \mathbf{M})$ \\
\hline MG-63 & 25 \\
\hline Saos-2 & 15 \\
\hline U-2 OS & 25 \\
\hline T1-73 & 25 \\
\hline $143 B$ & 30 \\
\hline HOS & 30 \\
\hline hFOB 1.19 & $>100$ \\
\hline
\end{tabular}

Following incubation with the primary antibodies, the membrane was washed with PBST and then incubated for $1 \mathrm{~h}$ with $5 \%$ dried skimmed milk powder, washed in PBST and incubated with horseradish peroxidase (HRP)-conjugated secondary antibodies (Bio-Rad Laboratories, Hercules, CA, USA). An enhanced chemiluminescence (ECL) detection kit (Amersham Pharmacia) was used for the analysis of the protein interactions.

\section{Statistical analysis}

The data were expressed as the mean \pm standard deviation (SD). The Student's t-test and one-way analysis of variance (ANOVA) were used for the comparison of the differences between the groups. For the purpose of relevance analysis of ordinal data,
A

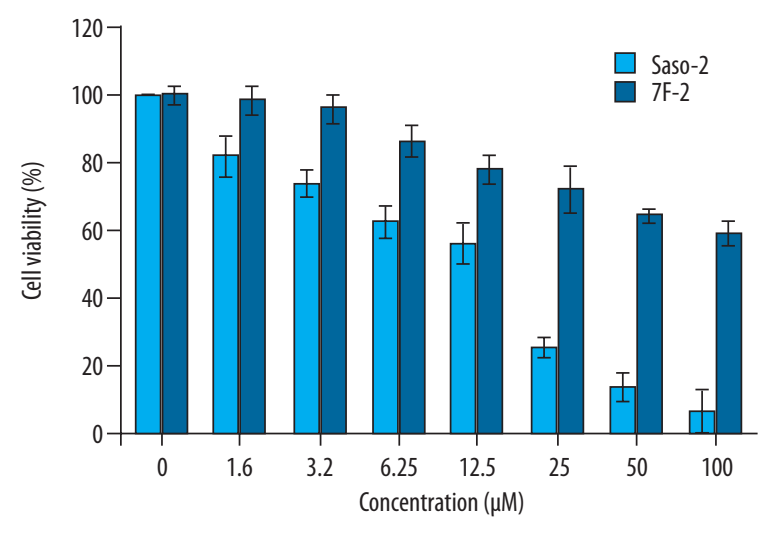

B
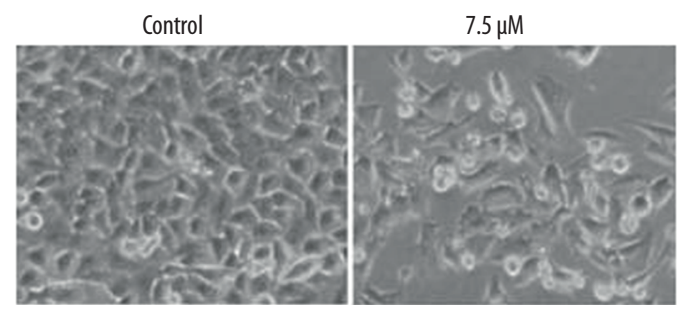

$15 \mu \mathrm{M}$

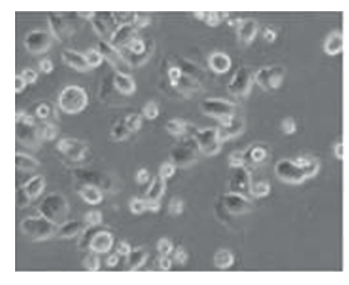

$30 \mu \mathrm{M}$

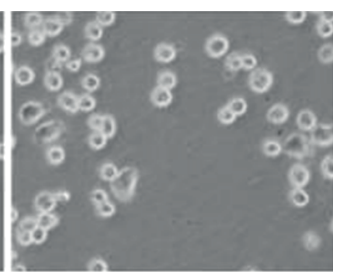

Figure 1. Effect of ludartin on the viability of Saos-2 osteosarcoma cells. (A) The effect of ludartin on osteosarcoma Saos-2 cells in the MTT assay. (B) The effect of ludartin of the morphology of Saos-2 cells. The experiments were performed in triplicate and the values presented as mean $\pm S D\left({ }^{*} P<0.05\right)$. 


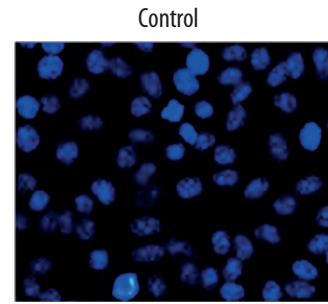

$15 \mu \mathrm{M}$

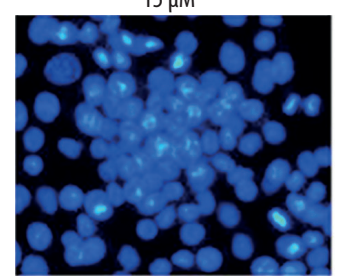

\section{$7.5 \mu \mathrm{M}$}

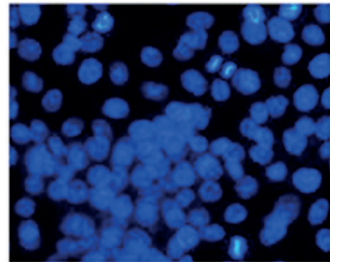

$30 \mu \mathrm{M}$

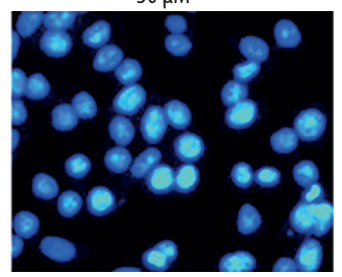

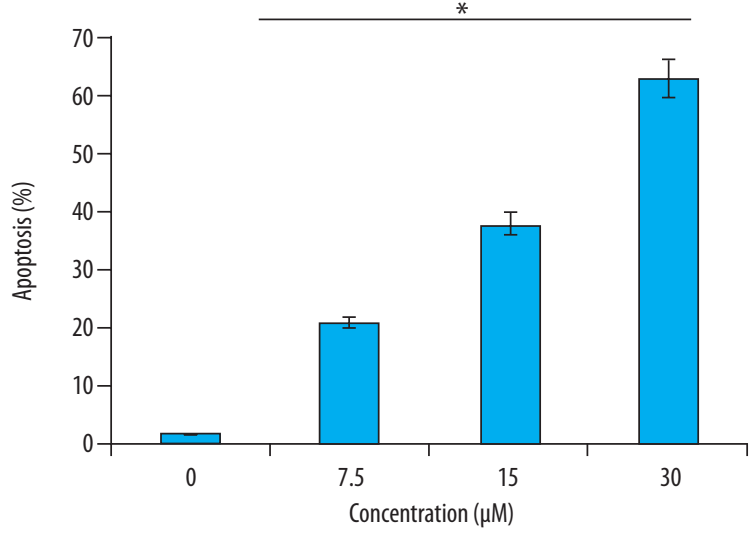

Figure 2. Ludartin triggered apoptosis in Saso-2 osteosarcoma cells. Apoptosis was studied by fluorescence-activated cell sorting (FACS) using 4', 6-diamidino-2-phenylindole (DAPI) nuclear staining. The experiments were performed in triplicate and the values presented as mean \pm SD $\left({ }^{*} \mathrm{P}<0.05\right)$.

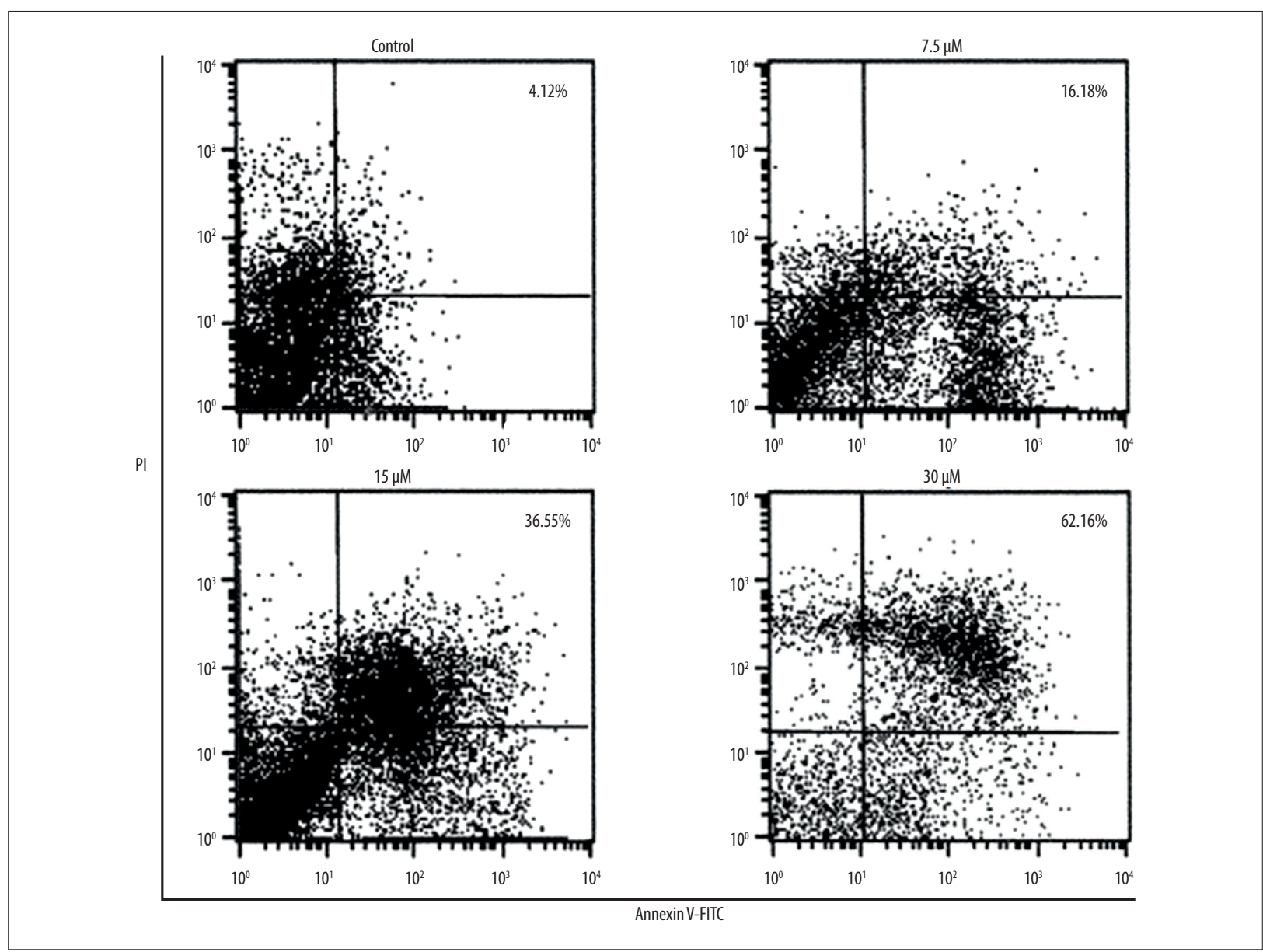

Figure 3. Estimation of apoptotic of Saos-2 osteosarcoma cell populations at indicated concentrations of ludartin. Annexin-V/propidium iodide (PI) staining. The experiments were performed in triplicate. 


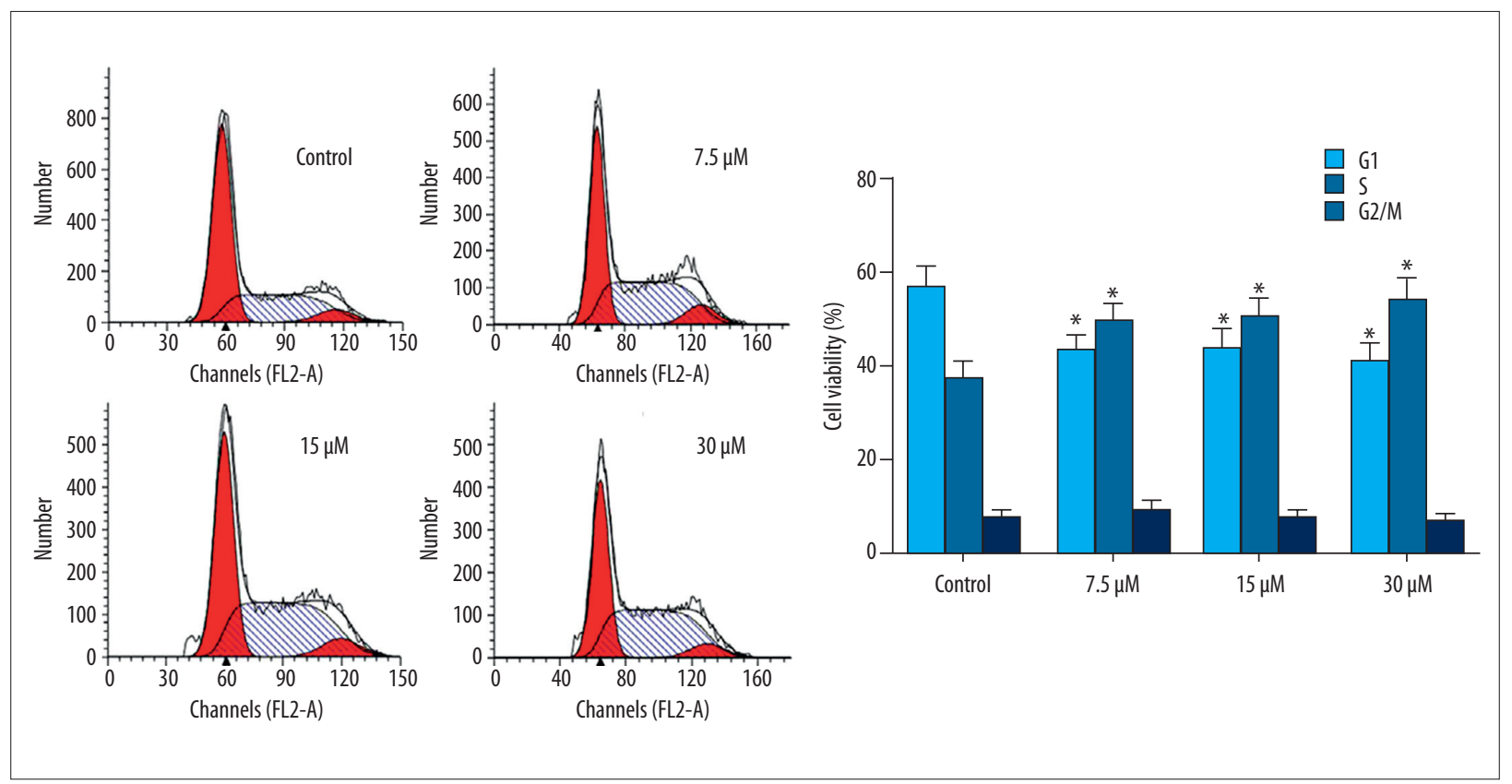

Figure 4. Ludartin treatment and G2/M cell cycle arrest in Saso-2 osteosarcoma cells as determined by flow cytometry. The experiments were performed in triplicate.

the cross chi-squared $\left(\chi^{2}\right)$ test was used. The values were considered statistically significant at $\mathrm{P}<0.05$.

\section{Results}

\section{Inhibition of cell viability of the Saos-2 osteosarcoma cells treated with ludartin}

The effect of increasing concentrations of ludartin $(0-100 \mu \mathrm{M})$ on the viability of a panel of osteosarcoma cells (MG-63 Saos-2 U-2OS, T1-73 143B, and HOS) and normal osteoblast cells (hFOB 1.19) was investigated by the MTT assay. The results showed a dose-dependent inhibition in the viability of all the osteosarcoma cell lines treated with ludartin (Table 1). The anti-proliferative effects of ludartin treatment were found to be minimal for the normal osteoblast cell line (hFOB 1.19). Thee osteosarcoma cell line that showed the greatest anti-proliferative response following treatment with ludartin was Saos-2 osteosarcoma cell line, which showed an $\mathrm{IC}_{50}$ at a dose of $15 \mu \mathrm{M}$ of ludartin (Figure 1A). Also, ludartin treatment induced distinctive morphological changes in the Saos-2 cells (Figure 1B)

\section{Induction of apoptosis in the Saos-2 osteosarcoma cells treated with ludartin}

Analysis of the Saos-2 osteosarcoma cells following fluorescence-activated cell sorting (FACS) analysis using 4',6-diamidino-2-phenylindole (DAPI) nuclear staining and Annexin-V/propidium iodide (PI) staining showed that ludartin treatment was associated with the induction of cell apoptosis (Figure 2). Also, the apoptotic effects of ludartin on Saos-2 cells were found to be dose-dependent. The apoptotic cell populations were determined by Annexin-V/PI staining, which showed that the apoptotic cells increased from $2 \%$ in untreated cells to up to $77 \%$ with ludartin concentrations of $30 \mu \mathrm{M}$ (Figure 3)

\section{Induction of G2/M cell cycle arrest in of the Saos-2 osteosarcoma cells treated with ludartin}

To further investigate other mechanisms that could cause ludartin-induced suppression of cell proliferation, the cell cycle was studied in Saos- 2 cells after ludartin treatment, using flow cytometry. Ludartin treatment resulted in the accumulation of Saos-2 osteosarcoma cells in the G2-phase by 24 hours following ludartin treatment (Figure 4).

Further immunoblotting was performed to determine the effects of ludartin on the expression of G2/M cell cycle-associated proteins including cyclin B1, Cdc25c, and Cdc2 in Saos-2 osteosarcoma cells. As shown in Figure 5A, treatment of Saos-2 osteosarcoma cells with ludartin resulted in a dose-dependent reduction in the expression of pCdc25c (Ser216), Cdc25c, pCdc2 (Tyr15) and Cdc2 in Saos-2 osteosarcoma cells. Also, treatment of Saos-2 osteosarcoma cells with ludartin for 48 hours resulted in reduced expression of cyclin B1. Ludartin treatment increased the expression of cyclin-dependent kinase inhibitor p21 (WAF1) or p21WAF1 in Saso-2 osteosarcoma cells (Figure 5B). However, ludartin treatment did not alter the expression of either p27 or p53 tumor suppressor proteins (Figure 5B). 


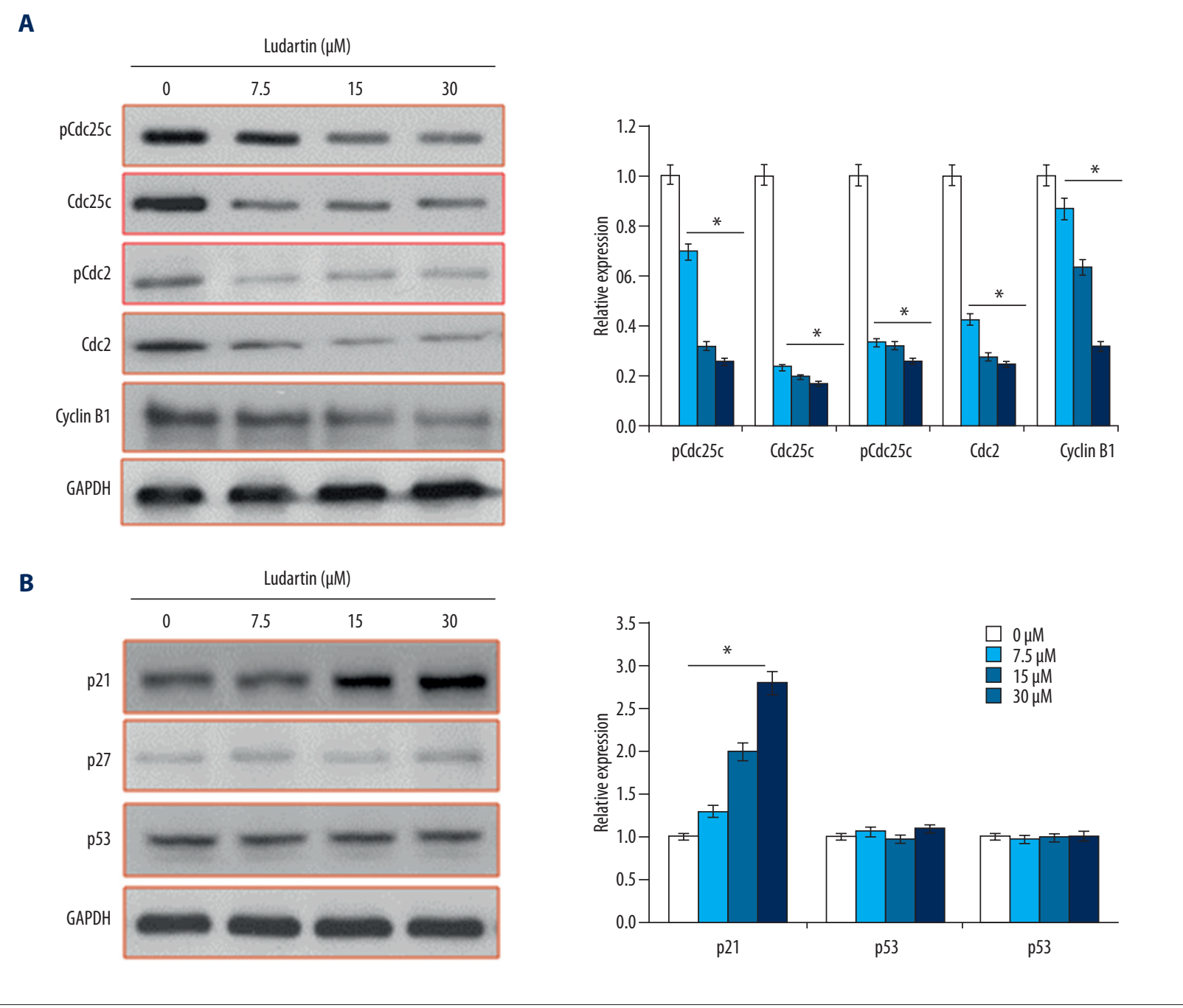

Figure 5. Western blots showing the effect of ludartin on cell cycle proteins in Saso-2 osteosarcoma cells. (A) Cdc25c, pCdc25c, Cdc2, pCdc2, p21WAFI, and cyclin B1, and (B) p21, p53, and p27. Ludartin treatment increased the expression of cyclin-dependent kinase inhibitor p21 (WAF1) or p21WAF1 in Saso-2 osteosarcoma cells but did not alter the expression of p27 or p53 tumor suppressor proteins. The experiments were performed in triplicate and the values presented as mean $\pm S D\left({ }^{*} P<0.05\right)$.

\section{Inhibition of cell migration and invasion of the Saos-2 osteosarcoma cells treated with ludartin}

Treatment of Saso-2 osteosarcoma cells with ludartin inhibited cell migration and invasion (Figure 6A, 6B). Moreover, the inhibition of migration and invasion of Saos-2 were also associated with down-regulation of MMP-2 and MMP-9 expression (Figure 6C).

\section{Discussion}

Worldwide, osteosarcoma is a significant cause of morbidity and mortality, and the current methods of treatment may not be effective and are associated with side effects [13]. Therefore, there is a need to identify new chemotherapeutic agents to treat osteosarcoma. The natural products used in Chinese herbal medicine might provide promising treatments for malignant disease, including osteosarcoma $[7,8]$. Therefore, the aim of this study was to investigate the anticancer effects of ludartin, a natural product, in a panel of osteosarcoma cell lines (MG-63, Saos-2, U-2OS, T1-73 143B, and HOS), and a normal osteoblast cell line (hFOB 1.19) in vitro. The findings of this study showed that ludartin treatment resulted in an anti-proliferative effect on all the osteosarcoma cell lines studied cell lines. However, the anti-proliferative effects were more pronounced on the osteosarcoma Saos- 2 cells $\left(I C_{50}\right.$ dose of $\left.15 \mu \mathrm{M}\right)$, and the least anti-proliferative effects were observed on the normal hFOB 1.19 osteoblast cells $\left(\mathrm{IC}_{50}\right.$ dose $\left.>100 \mu \mathrm{M}\right)$. 

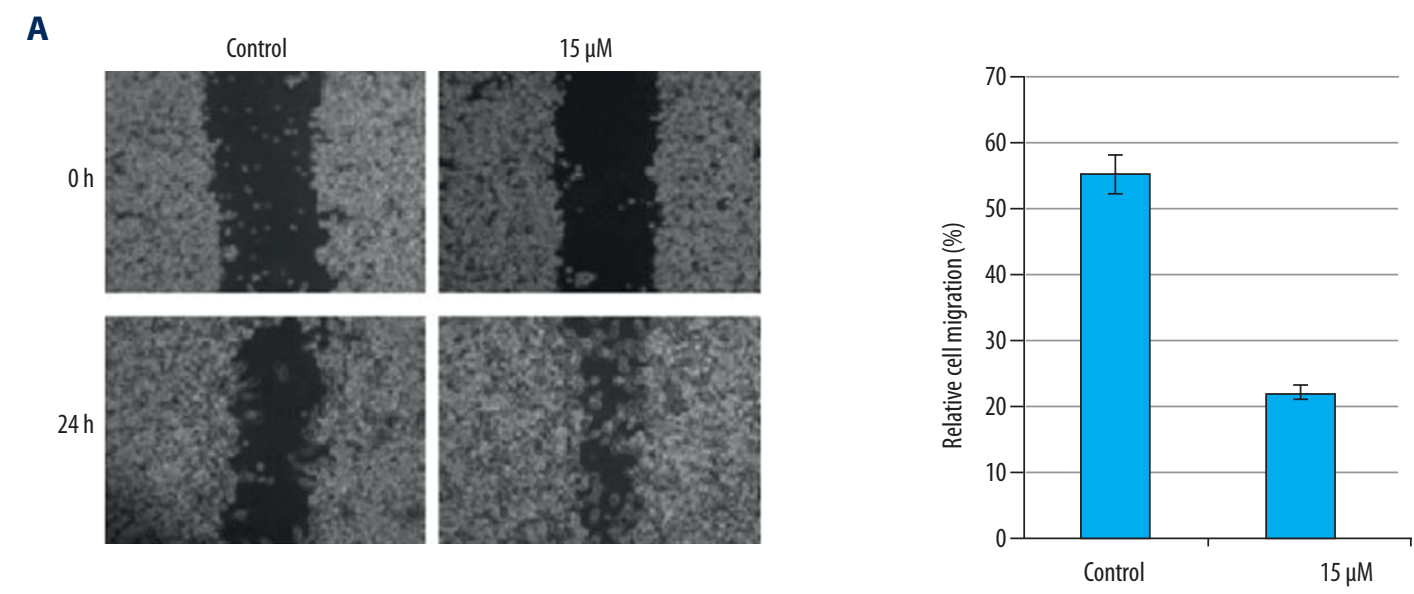

B
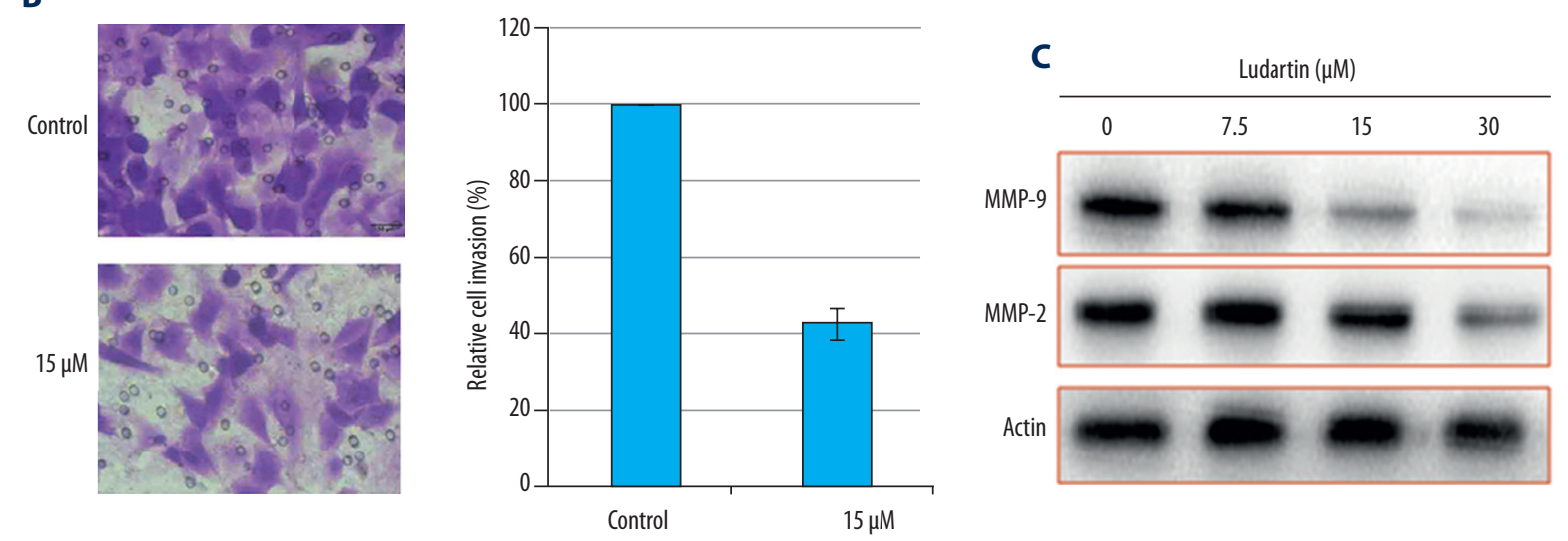

Figure 6. Effect of ludartin at the $\mathrm{IC}_{50}$ of the Saso-2 osteosarcoma cells. (A) Cell migration of Saso-2 osteosarcoma cells. (B) Cell invasion of Saso-2 osteosarcoma cells. (C) Expression of MMP-2 and 9 by Saso-2 osteosarcoma cells. The experiments were performed in triplicate and the values presented as mean $\pm \mathrm{SD}\left({ }^{*} \mathrm{P}<0.05\right)$.

The findings of the present study are supported by those of previously published studies, which have shown that ludartin treatment inhibited the proliferation and metastasis of ovarian cancer cells [9]. Also, in the present study, the anti-proliferative effects ludartin treatment on the Saos-2 osteosarcoma cells studied in vitro were also associated with changes in cell morphology, which supports that ludartin selectively targeted osteosarcoma cells in this study. Given the initial findings in the Saos-2 osteosarcoma cells in the present study, further experiments were performed on these cells.

In this study, to evaluate the possible mechanism of the antiproliferative effects of ludartin treatment on Saos-2 osteosarcoma cells, the effects on cell apoptosis were studied by fluorescence-activated cell sorting (FACS) using 4',6-diamidino2-phenylindole (DAPI) nuclear staining and Annexin-V/propidium iodide (PI) staining, which confirmed that ludartin induced apoptosis. Many anti-cancer drugs exert their therapeutic activity by triggering apoptosis of rapidly proliferating cancer cells, including the chemotherapeutic agent cisplatin [14,15]. Also, the resistance of the cancer cells to drugs is in part due to their capacity to escape apoptosis [16]. In the present study, the impact of ludartin on the phases of the cell cycle of the Saos-2 osteosarcoma cells were studied and the findings were that ludartin treatment induced G2/M cell cycle arrest, indicating that cells at the G2/M phase could not progress and undergo mitosis due to the inhibition of proteins such as cyclin B1, Cdc25C, and Cdc2. Also, p21WAF1 is an important inhibitor of cell cycle progression, and p21WAF1 expression was shown to be increased in Saos-2 osteosarcoma cells treated with ludartin in the present study [17]. There have been published findings that indicate that upregulation of p21WAF1 inhibits the expression of the $\mathrm{Cdc} 2 /$ cyclin B1 complex, which when activated triggers entry of the cell into mitosis [18].

Therefore, from the findings of this preliminary in vitro study, the treatment of Saos-2 osteosarcoma cells with ludartin inhibited the expression of cyclin B1, Cdc25C, and Cdc2 and 
increased the protein expression levels of p21WAF1, had no significant effect on the expression of p27 or p53. These findings might indicate that ludartin treatment induced the expression of p21WAF1, which in turn triggered G2/M-phase arrest. Cell migration and invasion is one of the important characteristic features cancer progression and metastasis [19]. Inhibition of cell migration and invasion prevents cancer metastasis and improves patient survival in vivo [19]. In the present study, treatment of Saos-2 osteosarcoma cells with ludartin inhibited cell migration and invasion in vitro. The findings of this preliminary study support the need for further studies to evaluate the effects of ludartin on other tumor cells lines in vitro together with an assessment of the effects of ludartin in vivo, to determine whether ludartin may prove to be a useful future chemotherapeutic agent.

\section{References:}

1. Mueller F, Fuchs B, Kaser-Hotz B: Comparative biology of human and canine osteosarcoma. Anticancer Res, 2007; 27: 155-64

2. Luetke A, Meyers PA, Lewis I, Juergens $\mathrm{H}$ : Osteosarcoma treatment - where do we stand? A state of the art review. Cancer Treat Rev, 2014; 40: 523-32

3. Isakoff MS, Bielack SS, Meltzer P, Gorlick R: Osteosarcoma: Current treatment and a collaborative pathway to success. J Clin Oncol, 2015; 33: 3029-35

4. ChangShui Y, WenBo W: Relationship between P15 gene mutation and formation and metastasis of malignant osteosarcoma. Med Sci Monit, 2016; 22: 656-61

5. Zhi C, Jingdong W, Qinghao G: Actein inhibits cell proliferation and migration in human osteosarcoma. Med Sci Monit, 2016; 22: 1609-16

6. Lin B, Huang D, Yu C et al: MicroRNA-184 modulates doxorubicin resistance in osteosarcoma cells by targeting BCL2L1. Med Sci Monit, 2016; 22: 1761-65

7. Das B, Satyalakshmi G: Natural products-based anticancer agents. MiniRev Org Chem, 2012; 9: 169-77

8. Da Rocha AB, Lopes RM, Schwartsmann G: Natural products in anticance therapy. Curr Opin Pharmacol, 2001; 1: 364-69

9. Bai L, Wang H, Zhang LY et al: Ludartin treatment exhibits promising inhibitory effect on the epithelial ovarian cancer growth and metastasis. Bangla J Pharmacol, 2016; 11: 646-51

10. Robles M, Aregullin M, West J, Rodriguez E: Recent studies on the zoopharmacognosy, pharmacology and neurotoxicology of sesquiterpene lactones. Planta Medica, 1995; 61: 199-203

\section{Conclusions}

The findings of this study showed that treatment of Saos-2 osteosarcoma cells in vitro with ludartin inhibited cell proliferation, cell migration, increased apoptosis of proliferating malignant cells, induced cell cycle arrest at the G2/M checkpoint, findings that were associated with increased expression of p21WAF1. Therefore, future in vivo studies are required to determine whether ludartin has a potential role as a lead molecule in the treatment of osteosarcoma and other types of malignancy.

11. Daniel B, DeCoster MA: Quantification of SPLA2-induced early and late apoptosis changes in neuronal cell cultures using combined TUNEL and DAPI staining. Brain Res Brain Res Protoc, 2004; 13: 144-50

12. Kao WT, Lin CY, Lee LT et al: Investigation of MMP-2 and-9 in a highly invasive A431 tumor cell sub-line selected from a Boyden chamber assay. Anticancer Res, 2008; 28: 2109-20

13. Glover J, Man TK, Barkauskas DA et al: Osteosarcoma enters a post genomic era with in silico opportunities: Generation of the High Dimensional Database for facilitating sarcoma biology research: A report from the Children's Oncology Group and the QuadW Foundation. PLoS One, 2017; 12: e0181204

14. Gonzalez VM, Fuertes MA, Alonso C, Perez JM. Is cisplatin-induced cell death always produced by apoptosis? Mol Pharmacol, 2001; 59: 657-63

15. Wang X, Martindale JL, Holbrook NJ: Requirement for ERK activation in cisplatin-induced apoptosis. J Biol Chem. 2000; 275: 39435-43

16. Tsuruo T, Naito M, Tomida A et al: Molecular targeting therapy of cancer Drug resistance, apoptosis and survival signal. Cancer Sci, 2003; 94(1): $15-21$

17. Somasundaram K, Zhang H, Zeng YX et al: Arrest of the cell cycle by the tumour-suppressor BRCA1 requires the CDK-inhibitor p21WAF1/CiPl. Nature, 1997; 389: 187-90

18. Winters ZE, Hunt NC, Bradburn MJ et al: Subcellular localisation of cyclin B, Cdc2 and p21 WAF1/CIP1 in breast cancer: Association with prognosis. Euro J Cancer, 2001; 37: 2405-12

19. Helbig G, Christopherson KW, Bhat-Nakshatri $P$ et al. NF- $\mathrm{KB}$ promotes breast cancer cell migration and metastasis by inducing the expression of the chemokine receptor CXCR4. J Biol Chem, 2003; 278(24): 21631-38 